\title{
Optimizing templates for finding trees in aerial photographs
}

\author{
Morten Larsen ${ }^{\mathrm{a}, *}$, Mats Rudemo ${ }^{\mathrm{b}, 1}$ \\ a Department of Mathematics and Physics, Royal Veterinary and Agricultural University, Thorvaldsensvej 40, 1871 Frederiksberg C, \\ Denmark \\ b Department of Mathematical Statistics, Chalmers University of Technology and Gothenburg University, 41296 Gothenburg, Sweden
}

Received 11 February 1998; received in revised form 9 August 1998

\begin{abstract}
Positions of individual trees in high-resolution aerial photographs are estimated by use of templates derived from an optical tree crown model and the geometry at image acquisition. The template is bounded by an ellipse, for which we find the optimal size, eccentricity and translation along one axis. Tree top positions are estimated as local maxima of the correlation function for the successively transposed template over the whole image. Performance is evaluated using three images from a thinning experiment in spruce. The templates are compared with empirical shapes obtained by averaging over the individual trees in the image. (c) 1998 Elsevier Science B.V. All rights reserved.
\end{abstract}

Keywords: Shape estimation; Optimal template; Tree crown template; Individual tree positions; Optical tree crown model

\section{Introduction}

Smoothing by convolution with isotropic Gaussian kernels was used to estimate the number of trees per hectare from high resolution aerial photographs of spruce stands in (Dralle and Rudemo, 1996) and to estimate individual tree positions in (Dralle and Rudemo, 1997). The method was found to perform satisfactorily for trees reasonably close to the nadir point in the photograph (Dralle, 1997).

An alternative method was studied in (Larsen and Rudemo, 1997) and (Larsen, 1997), where an optical tree crown model introduced in (Pollock, 1994a,b, 1996) was extended. The method gives a template for an individual tree adapted to the ge-

\footnotetext{
${ }^{*}$ Corresponding author. E-mail: ml@dina.kvl.dk.

${ }^{1}$ E-mail: rudemo@math.chalmers.se.
}

ometry at image acquisition. Tree top positions are estimated as local maxima of the correlation function for the successively transposed template over the whole image. The method was found to improve the kernel smoothing method for off-nadir viewing angles. Some such angles might actually give the most accurate tree top position estimates for spruce and other trees with similar morphology (such as fir).

For the kernel smoothing method a crucial issue is the choice of the shape and size of the kernel, and one main issue in (Dralle and Rudemo, 1996) was to introduce and study a method for optimal size (bandwidth) estimation for an isotropic kernel.

For the method based on the optical tree crown model there is a similar problem on estimation of the optimal size and shape of the boundary within which the single tree template is valid. In the present paper we study estimation of 
the optimal size and shape of an elliptic boundary for this template and the optimal placement along the tree trunk of the boundary relative to the tree top.

Performance is evaluated by use of three images from a thinning experiment in Norway spruce, corresponding to sidelighted, backlighted and frontlighted trees, respectively. The templates for these three geometries are also compared with the empirical shapes obtained by averaging over the individual trees in the image.

\section{The optical model}

We use the optical tree model from (Larsen, 1997). By including reflection from two types of scatterers: "needles" and "branches" this model extends the model in (Larsen and Rudemo, 1997), which in turn extends the model in (Pollock, 1994a,b, 1996) by including background to the modelled tree crown. The basic elements are a single tree crown, a "ground" plane, light sources and a camera.

\subsection{Light sources and camera}

Only the sun and the sky are considered. The diffuse illumination from the ground and from interreflections between trees is ignored. The sun is modelled as a collimated beam of light, and the direction to the sun is computed from the time and place of image acquisition.

The clear sky is modelled as a number of discrete collimated light beams emanating from directions evenly distributed over the sky hemisphere as suggested in (Woodham and Gray, 1987). As an approximation we use a uniform distribution for the distribution of light in the sky, and we do not take the shadowing by neighbouring trees into account. The power of the sun relative to the clear sky is set to 1.6 , based on light-shadow contrasts in the images used.

The camera is assumed to be a pinhole camera. Its location, resolution, the direction of the optical axis and the "up" direction in the image plane are computed from the image rectification parameters (Dralle, 1997).

\subsection{Tree crown shape}

The basic tree crown shape is modelled as a generalised ellipsoid that in $(x, y, z)$ coordinates has the surface

$\frac{\left(z^{2}\right)^{n / 2}}{a^{n}}+\frac{\left(x^{2}+y^{2}\right)^{n / 2}}{b^{n}}=1$,

where $z$ is the vertical axis, the "centre" of the tree crown is at the origin, $a$ is half the length of the ellipsoid, $b$ is half the width and $n$ is a shape parameter; in the experiment described below we use $a=17.7 \mathrm{~m}, b=2.84 \mathrm{~m}$ and $n=1.6$ as in (Larsen, 1997).

The crown ellipsoid is truncated downwards by a horizontal plane intersecting the vertical axis at $z=z_{\text {base }},-a \leqslant z_{\text {base }}<a$. This is especially relevant for spruce or fir, where in this work only the upper half of the ellipsoid is used.

\subsection{Crown and light interaction}

The interior of the tree crown is assumed to contain randomly oriented scatterers (needles, twigs, etc.). The scatterers can be of several (here two) different types $t \in T$ having different optical properties and distributions. Their density within the tree crown envelope is assumed proportional to a function $f^{(t)}(\boldsymbol{r}) \geqslant 0, \boldsymbol{r}=(x, y, z)$, such that the proportion of a ray of light that will pass without interaction (be transmitted) through a volume containing scatterers is given by

$p_{\text {tr }}(L)=\exp \left(-\int \sum_{L \in T} f^{(t)}(\boldsymbol{r}) \mathrm{d} w\right)$

(Beer-Lambert's Law). The integral is over the path $L$ of the ray through the volume, with $\boldsymbol{r}=\boldsymbol{r}(w)$. The light not transmitted will be either absorbed or reflected by the scatterers. Only single reflections are modelled, so the proportion $p_{\text {refl }}(\boldsymbol{r})$ of non-transmitted light isotropically reflected in a small volume around $\boldsymbol{r}$ is equal to

$p_{\text {refl }}(\boldsymbol{r})=\frac{\sum_{t \in T} p_{\text {refif }} f^{(t)}(\boldsymbol{r}),}{\sum_{t \in T} f^{(t)}(\boldsymbol{r}),}$

where $p_{\text {refl }}^{(t)}$ is the reflectivity factor for the scatterers of type $t$. 
If $C$ is the path of a camera ray through the tree crown and $P_{i}$ is the power per unit area perpendicular to the beam direction of light source $i$ then the power per unit area $P(C)$ of the light reflected by the tree crown in the direction of that camera ray is

$$
\begin{aligned}
P(C)= & K_{\text {refl }} \sum_{i \in \text { lights }} P_{i} R(i, C), \\
R(i, C)= & \int_{C} p_{\mathrm{tr}}\left(C_{s}(\boldsymbol{r})\right) p_{\mathrm{tr}}\left(L_{i}(\boldsymbol{r})\right) p_{\mathrm{refl}}(\boldsymbol{r}) \\
& \left(\sum_{t \in T} f^{(t)}(\boldsymbol{r})\right)\left|\frac{\mathrm{d} \boldsymbol{r}}{\mathrm{d} w}\right| \mathrm{d} w
\end{aligned}
$$

where $K_{\text {refl }}$ is a constant giving the proportion of the total reflected light which is reflected towards the camera, $C_{s}(\boldsymbol{r})$ is the subpath of $C$ towards the camera from the point $\boldsymbol{r}=\boldsymbol{r}(w)$ on $C$ and $L_{i}(\boldsymbol{r})$ is the path through the tree crown from $r$ towards light source $i$, see Fig. 1.

\subsection{Crown composition}

As in (Larsen, 1997), where more details are given, we assume that the crown of a Norway spruce consists of two types of scatterers: needles

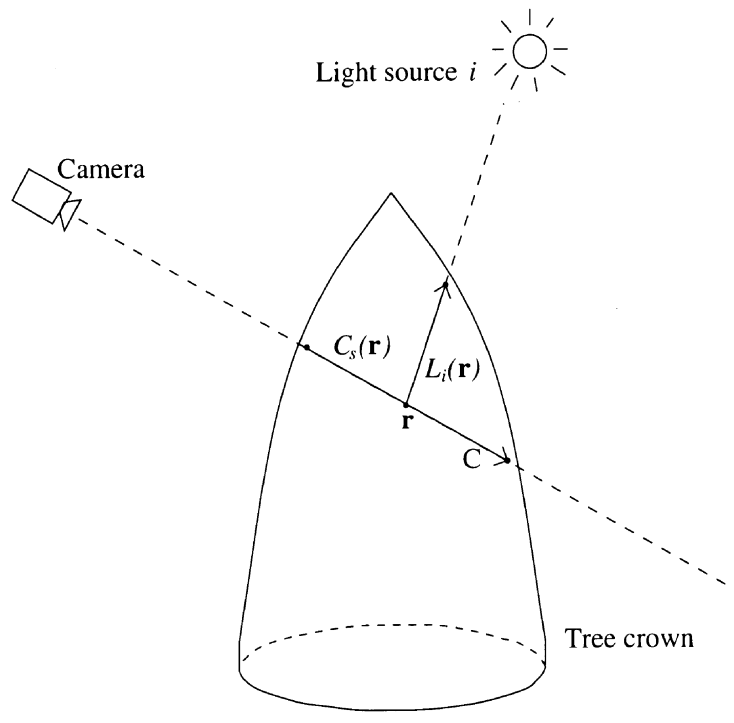

Fig. 1. Illustration of the elements in Eq. (5). and branches. The density of needles within the crown is given by

$f^{(\text {needles })}(u)= \begin{cases}c_{0}\left(\frac{u}{u_{\mathrm{d}}}\right)^{c_{1}}\left(1-\frac{u}{u_{\mathrm{d}}}\right)^{c_{2}} & 0 \leqslant u \leqslant u_{\mathrm{d}}, \\ 0 & \text { otherwise },\end{cases}$

where $u$ is the horizontal distance from the closest point on the tree crown envelope and $u_{\mathrm{d}}$ is the maximum depth of live needles within the tree crown. The parameters $c_{0}>0, c_{1}>0$ and $c_{2}>0$ are determined from $u_{\mathrm{d}}$ together with the depth of maximum needle density $u_{\max }$, the average needle density $f_{\text {avg }}$ and the maximum needle density $f_{\max }$ (Pollock, 1996). In the present work the values $u_{\mathrm{d}}=2 \mathrm{~m}, \quad u_{\max }=0.5 \mathrm{~m}, f_{\text {avg }}=1$ and $f_{\max }=2$. These values are based on information found in (Oker-Blom and Kellomäki, 1982; Koppel and Oja, 1984). The panchromatic reflection factor $p_{\text {refl }}^{\text {(nedles) }}$ is set to $9 \%$ based on (Williams, 1991).

The model including needles as the only scatterer was found to be unable to reproduce the sharp contrast between light and shadow observable quite near the top of spruce trees backlighted by the sun. This motivated the introduction of something to model the trunk and branches.

The density function for the scatterers corresponding to the branches is set to

$f^{(\mathrm{br})}(v)=c_{3} \exp \left(-c_{4} v^{c_{5}}\right)$,

where $v$ is the horizontal distance from the vertical axis of the tree relative to the radius of the tree crown envelope, $0 \leqslant v \leqslant 1$. The parameters $c_{3}>0$, $c_{4}>0$ and $c_{5}>0$ are computed from the desired values of $f^{(\mathrm{br})}(0.1), f^{(\mathrm{br})}(0.3)$ and $f^{(\mathrm{br})}(0.6)$, corresponding roughly to trunk, large branches and small branches, and the densities 0.5, 0.08 and 0.02 respectively were chosen. The panchromatic reflection factor $p_{\text {refl }}^{\text {(br) }}$ was set to $0 \%$ to obtain a pure shadowing effect.

\subsection{The "ground" plane}

A "ground" plane is included in the model to provide a background for the modelled tree crown, thereby giving a brightness edge at the crown boundary and, for some angles, allowing the modelled tree to cast a shadow in the ray-traced template. With properly chosen reflectance parameters this is a simple way to describe the average effect 
of the complex background of neighbouring trees partly in light and partly in shadow.

The "ground" plane is a horizontal surface that reflects and absorbs light. The reflectance function used is the Minnaert reflectance function, cf. (Woodham and Gray, 1987), where the power $P_{g}$ of the light reflected in the direction of the camera can be computed from the incident angle $\theta_{i}$ of the light from the light source $i$ and the exit angle $\theta_{c}$ of the light reflected towards the camera:

$P_{g}(i)=P_{i} \rho \frac{k+1}{2 \pi} \cos ^{k}\left(\theta_{i}\right) \cos ^{k-1}\left(\theta_{c}\right)$,

where $\rho$ is the reflectance factor and $0 \leqslant k \leqslant 1$ is a constant characterising the surface (the value $k=1$ chosen in this work yields a Lambertian surface). The reflectance factor $\rho$ should be chosen to yield a "ground" plane reflection corresponding to the average background for tree crowns in the actual image. The values $\rho=0.014,0.017$ and 0.020 were used for the three images " 120 ", "124" and "144", respectively (see Section 4 for an explanation of the image numbers).

\section{Local correlation maxima}

The geometric-optical model is used to produce a template image, bounded by an ellipse, of a single tree as it would look if it was placed in the centre of the stand. For near-nadir views or for large stands it may be necessary to produce several templates for different positions within the stand. The bounding ellipse has one axis along the tree trunk projection as seen from the camera. The size and shape (eccentricity) of the ellipse and the position of the ellipse centre along the tree trunk projection are three parameters that we optimize.

For each image pixel within the stand the local correlation between the template and the image is computed. The positive local maxima of the resulting correlation image correspond to the locations that (locally) resemble the template most closely and hence adjusted for the position of the model tree top within the template are candidates for tree top positions.

Usually there will be too many candidate positions, some where a tree top is detected at multiple nearby locations and some corresponding to large branches, artifacts due to the overlapping of tree crowns or minor variations in the image intensity in low-contrast parts of the image. Several possible strategies for retaining as many of the true tree top positions as possible while removing as many false positions as possible are examined in (Larsen and Rudemo, 1997).

The approximate number of trees in the stand may be known in advance, for example from automatic estimation methods such as the method described in (Dralle and Rudemo, 1996). In this case the number of candidates to be retained is equal to the expected number of trees, $N_{\text {trees }}$.

In this work the $N_{\text {trees }}$ candidates corresponding to the highest correlation values were selected, subject to the restriction that no selected candidate could be closer to a selected candidate of higher correlation value than a certain distance $d_{\min }$, measured in a horizontal plane placed at average tree top level.

A greedy algorithm selecting candidates in order of descending correlation and omitting any candidates too close to already selected candidates was used. The parameter $d_{\min }$ was set equal to $1 \mathrm{~m}$.

\section{Experiment}

Images showing a sub-plot of a thinning experiment in Norway spruce is used for testing the algorithm. The images were acquired about $40 \mathrm{~km}$ northwest of Copenhagen from an altitude of 560 $\mathrm{m}$ in clear-sky conditions in the morning of 4 August 1994. At image acquisition the trees were 48 years old. The average tree height was $22.7 \mathrm{~m}$ and the stem number was 386 trees per hectare. There were $N_{\text {trees }}=171$ trees visible from the air in the sub-plot. The digital image was scanned from contact prints and the final image resolution obtained was about $15 \mathrm{~cm}$ per pixel; see (Dralle and Rudemo, 1996) for further details on the image material.

Three images were selected from those available: one where the trees were sidelighted by the sun (image "120"), one where they were backlighted (image "124") and one where the trees were 
frontlighted, i.e. the sun was behind the camera (image "144"). See Figs. 2-4.

\subsection{Matching and parameter optimization}

Tree base positions measured in the field were extrapolated to estimated average tree top height (Dralle and Rudemo, 1997) and superimposed on the images to yield an initial estimate of the true tree top positions. These positions were adjusted by manual inspection of the images to correct for the errors introduced by errors in the tree height estimates, variations due to wind, and imprecisions in the image rectifications. The resulting tree top positions coincide with the centres of the circles plotted in Figs. 2-4. They were used as "ground truth" in the computations described below.

In this paper we will for each image consider two planes, the rendered image plane, as seen in the left parts of Figs. 2-4, and a parallel model tree top plane through the top of a model tree placed at the centre of the stand. Points in one plane are projected through the (pinhole) camera focal point to points in the other and as the planes are parallel any shape will simply be scaled through this projection. When we in the following refer to distances in meters we refer to distances measured in the model tree top plane.

Three parameters, size $r$, shape $s$ and translation $t$, for the ellipse bounding the tree crown template were optimized by minimization of a suitably chosen penalty function. Here $r$ is the radius of a circle (in the model tree top plane) with the same area as the ellipse, and $s$ is the width to length ratio of the ellipse. Here "length" is the dimension of the ellipse along the projected image of the tree trunk, e.g. from lower left to upper right in the small right upper image in Fig. 2, and "width" is the dimension orthogonal to the tree trunk. Further, $t$ is the translation in $r$-units of the centre of the ellipse downwards from the tree top along the tree trunk, i.e. $t r$ is the downwards translation in meters.
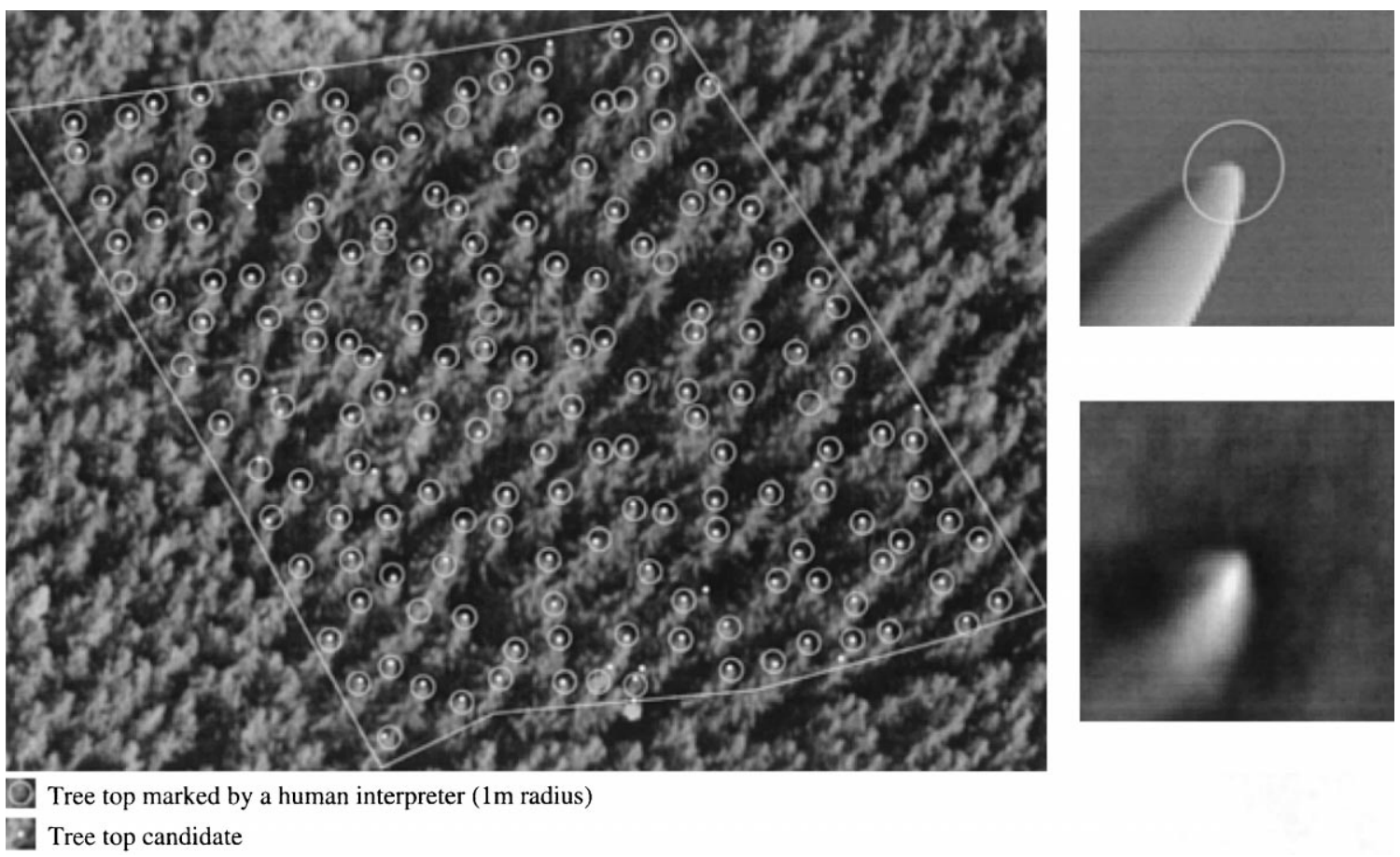

Fig. 2. The sidelighted image " 120 " with the 171 tree tops as manually marked and automatically estimated, the corresponding single reflection optical model with the optimal boundary (right upper) and the empirical average shape (right lower). 

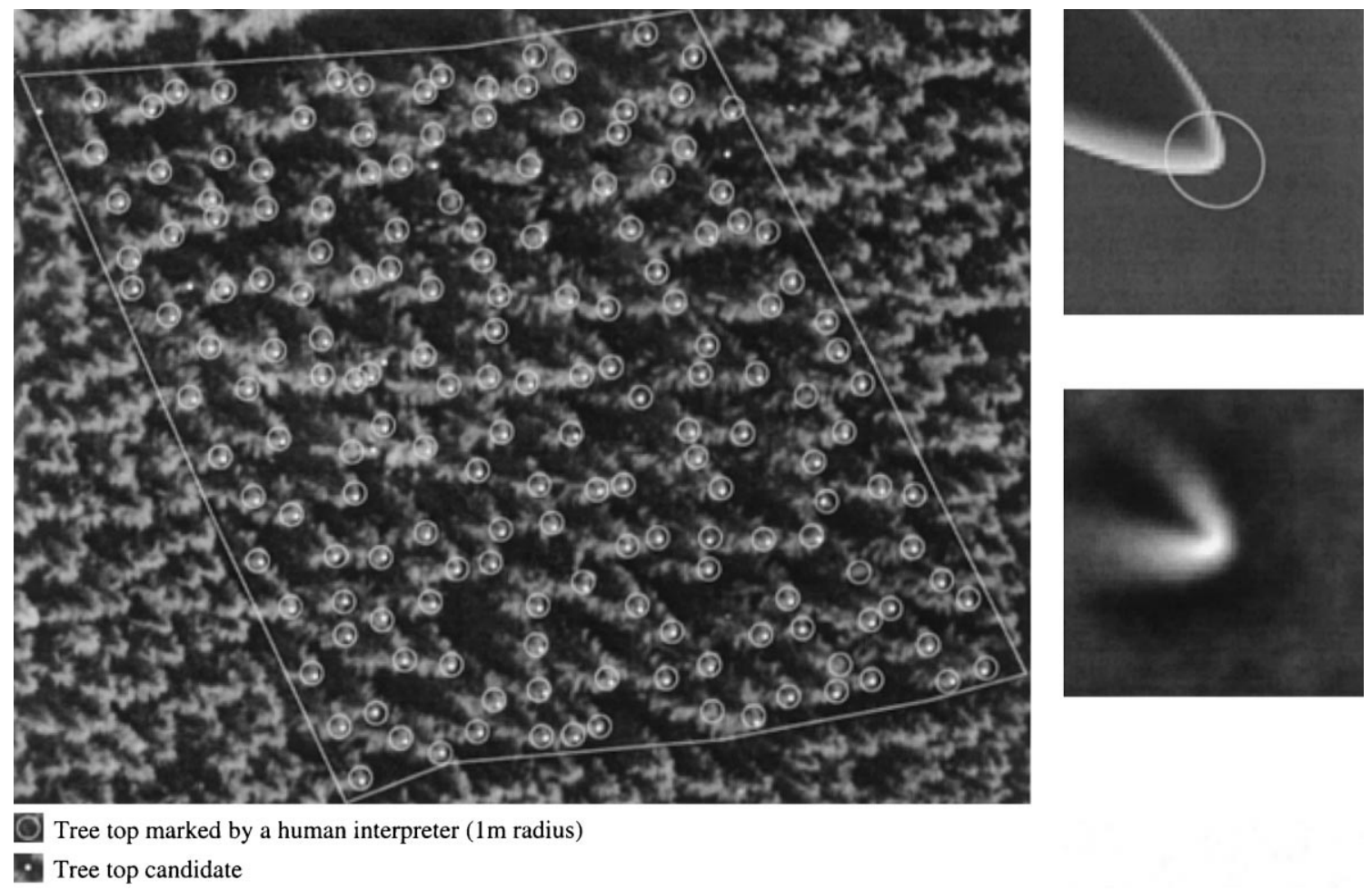

Fig. 3. The backlighted image " 124 " with the 171 tree tops as manually marked and automatically estimated, the corresponding single reflection optical model with the optimal boundary (right upper) and the empirical average shape (right lower).

A simple procedure was used to match a given set of tree top candidates with the "ground truth": Pairs of positions from the two sets were found in order of increasing error distance such that each position in each set was used only once. This procedure was stopped when the error distance in the next match would be greater than or equal to $d_{\max }=1 \mathrm{~m}$ (this value is equal to what was chosen for the threshold $d_{\min }$ ). All trees not matched at this point were declared "unmatched".

The penalty measure used to compare the set of tree top candidates with the "ground truth" data for a single image was a modified standard error measure computed as follows:

$\mathrm{SE}^{*}=\sqrt{\frac{\sum_{i \in \text { matched }}\left|\boldsymbol{x}_{i}-\overline{\boldsymbol{x}}\right|^{2}+n_{\text {unmatched }} d_{\text {max }}^{2}}{n_{\text {matched }}+n_{\text {unmatched }}}}$,

where the sum is taken over all matched tree tops, $\boldsymbol{x}_{i}$ is the error vector for matched tree top $i,|\cdot|$ is the Euclidian distance, $\overline{\boldsymbol{x}}$ is the average error vector for all matched tree tops, $n_{\text {matched }}$ and $n_{\text {unmatched }}$ are the number of matched and unmatched tree tops, respectively, and $d_{\max }$ is the maximum error accepted in the matching algorithm as outlined above. This penalty measure takes into account any systematic differences between what the human interpreter perceives as the tree top and what is the tree top according to the method used to produce the given set of candidates.

To optimize the parameters for the images used in the present study, the arithmetic average $\overline{\mathrm{SE}^{*}}(r, s, t)$ of the standard errors (9) for the three individual images was used as penalty function. This penalty function was computed for $(r, s, t) \in G$, where $G$ is a suitable grid of parameter combinations. Then, for a close grid $G_{\text {close }}$ around the minimizing parameter combination, a second degree polynomial in $r, s$ and $t$ was fitted and minimized. 

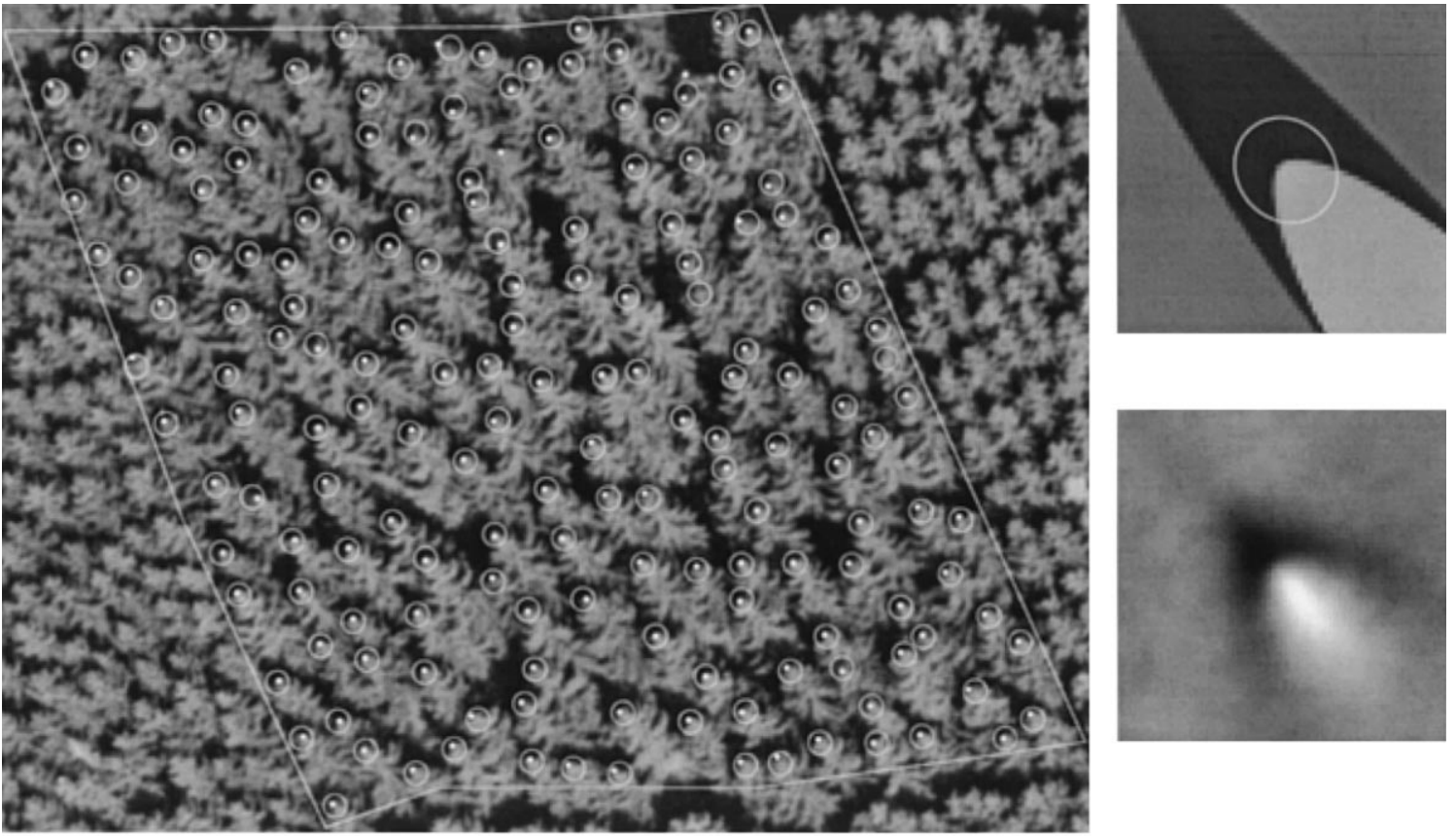

Tree top marked by a human interpreter (1m radius)

Tree top candidate

Fig. 4. The frontlighted image " 144 " with the 171 tree tops as manually marked and automatically estimated, the corresponding single reflection optical model with the optimal boundary (right upper) and the empirical average shape (right lower).

\subsection{Results}

As a first step in estimating the optimal parameter values the grid

$$
\begin{aligned}
(r, s, t) \in G= & \{1.0,1.5, \ldots, 4.0\} \\
& \times\{0.5,0.6, \ldots, 1.3\} \\
& \times\{-1.0,-0.5, \ldots, 2.0\}
\end{aligned}
$$

was used, and the penalty minimum was obtained for $r=1.5 \mathrm{~m}, s=1.0$ and $t=0.0$. In a second step the grid

$$
\begin{aligned}
(r, s, t) \in G_{\text {close }}= & \{1.25,1.30, \ldots, 1.75\} \\
& \times\{0.80,0.85, \ldots, 1.20\} \\
& \times\{-0.50,-0.45, \ldots, 0.50\}
\end{aligned}
$$

was investigated, and a second degree polynomial was fitted. The minimum of the penalty function was obtained for

$r=1.5 \mathrm{~m}, \quad s=0.9, \quad t=0.2$,

which thus gave the estimated optimal parameters.
With these optimal parameters the numbers of missed trees, which in the present setup means the number of trees not found within $1 \mathrm{~m}$ of the estimate found by a human interpreter, are given in Table 1 for the three images together with the standard error in estimation of the tree top position.

The tree top estimates for the optimizing parameter combination are shown in the left parts of Figs. 2-4 together with the tree tops found by a human interpreter. The corresponding templates are shown in the right upper parts of these figures,

Table 1

Number of missed trees for the three images

\begin{tabular}{lrrr}
\hline & \multicolumn{3}{l}{ Image } \\
\cline { 2 - 4 } & 120 & 124 & 144 \\
\hline Total number of trees & 171 & 171 & 171 \\
Number of missed trees & 15 & 6 & 3 \\
Percent of trees found & 91 & 96 & 98 \\
Standard error in cm (matched only) & 27 & 24 & 28 \\
Modified standard error (9) in cm & 39 & 30 & 31 \\
\hline
\end{tabular}


and in the right lower parts are shown empirical average shapes obtained by averaging the pixel values around the tree top positions found by the human interpreter. The grey level distributions in the images with the empirical averages in Figs. 2-4 have been stretched to enhance contrasts.

The scales in Figs. 2-4 are identical for the two small images in the right parts of the figures. To compare scales between the right and left parts in the figures, one may note that the circles around tree tops in the left parts have radii $1 \mathrm{~m}$, and the ellipses in the upper right parts have areas identical to the area of a circle of radius $r=1.5 \mathrm{~m}$.

Fig. 5 shows sensitivity functions, i.e. penalty functions (9) plotted for marginal variation of the three parameters around the global minimum. The figure shows results both for the three individual images and for the arithmetic average over the images.

\section{Discussion}

A recent trend in nonparametric function and density estimation is to use local modelling such as local polynomial fitting (Fan and Gijbels, 1996) and local likelihood estimation (Tibshirani and Hastie, 1987). Under some conditions these methods have better asymptotic properties than kernel smoothing methods. The template method for finding tree tops (or uniform objects in other spatial point processes) studied in the present paper seems to have similar advantages compared to the kernel smoothing method (Dralle and Rudemo, 1996, 1997).

The results presented in the present paper with an estimated standard error for the tree top position estimates of the order $25-30 \mathrm{~cm}$ and a recovery percent of $91-98 \%$ are quite satisfactory. Further improvement should be possible by combining results from several images by stereo methods.

Concerning the global optimum we were astonished to find the optimal size of the bounding ellipse to be so small, to find the optimal shape to be so close to a circle (we expected that the ellipse should be eccentric with a smaller width), and to find the optimal placement of the ellipse centre to
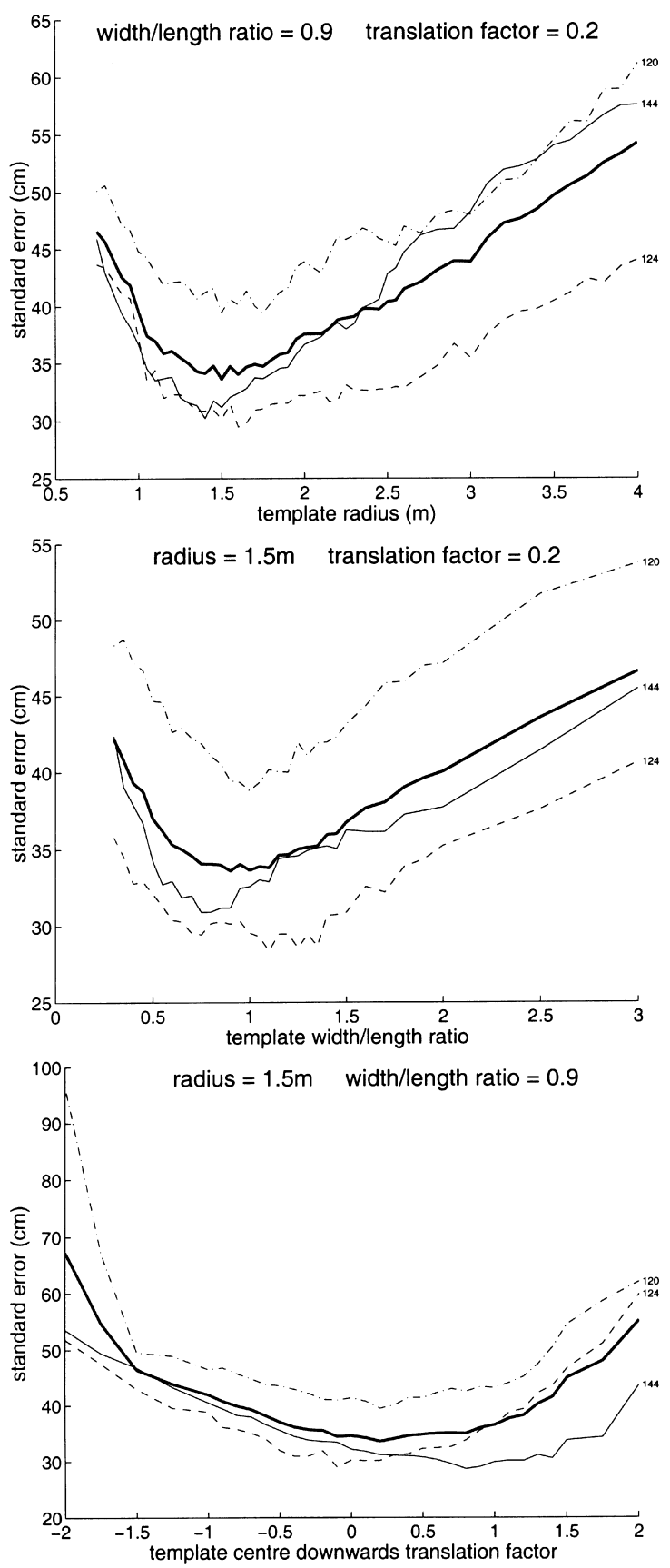

Fig. 5. Sensitivity functions, i.e. modified standard errors (9), of the optimal tree crown boundary with respect to variations in size (upper diagram), eccentricity (middle diagram) and translation along tree trunk (lower diagram). Diagrams are given both for the three individual images and for their average (the bold curve). 
be so close to the tree top (we expected it to be translated downwards).

Looking at Fig. 5 we see that the global optimal size, shape and placement of the bounding ellipse are generally quite good, although we could get some improvement by adapting the ellipse geometry to the individual images. In particular, for image 144 we could improve the ellipse by making it less wide and by sliding it further downwards along the tree trunk. For a near optimal all-round performance using only one parameter to determine the match window bounds one could on the other hand choose to have a simple circular match window centered at the model tree top, leaving only the match window size to be chosen for each stand.

Using a larger set of images and tree densities (we have here only studied the subplot with very heavy thinning) it should be possible to find how the optimal bounding ellipse should vary with the geometry at image acquisition and the tree density. With respect to tree density we expect that the curve giving the optimal size of the ellipse as a function of tree density should be essentially parallel to the curve that can be obtained by inverting the external curve in Fig. 7 in (Dralle and Rudemo, 1996). We expect that for Norway Spruce we can obtain good tree top identification results for tree densities which allow at least an average of 50 visible pixels per tree crown. This is because the conelike shape near the top of these trees assures that most "background" pixels will map to the shaded lower parts of the canopy which for moderate sun elevation angles are in good contrast to the sun-lit tree tops. For more closed canopies such as in deciduous forest the method will have to rely almost solely on the self-shading properties of the individual crown and can therefore not be expected to show the same performance.

Comparing the optimal templates for the single reflection model with the empirical averages in the right parts of Figs. 2-4 we find that the general appearance seems convincing. However, due to different sizes and varying close neighbourhoods of the individual trees the edges between different regions in the templates are smoothed in the empirical averages. Perhaps one could modify our templates by a corresponding minor blurring, but we are uncertain of whether this would yield improved performance.

\section{Conclusions}

The optimal shape and placement of the bounding ellipse for a tree template used to find tree positions in high resolution aerial photos have been estimated for a plantation with Norway spruce with heavy thinning. The optimal parameters for this ellipse seem fairly stable with respect to variations in the geometry at image acquisition. A comparison with empirical average shapes for three images with varying acquisition geometry shows a good general agreement with the theoretical templates.

\section{Acknowledgements}

The research reported in this paper was supported by the Danish Agricultural and Veterinary Research Council through Dina, Danish Informatics Network in Agricultural Sciences. The main part of the work was performed while Morten Larsen visited the Computational Vision and Active Perception laboratory (CVAP) at the Royal Institute of Technology in Stockholm, Sweden. We are further indebted to Kim Dralle, the Danish Forest and Landscape Research Institute, for providing image rectification and tree position data.

\section{References}

Dralle, K., 1997. Locating trees by digital image processing of aerial photos. Ph.D. Thesis, Royal Veterinary and Agricultural University of Denmark, Frederiksberg, Denmark, Dina Research Report No. 58.

Dralle, K., Rudemo, M., 1996. Stem number estimation by kernel smoothing in aerial photos. Canadian Journal of Forest Research 26, 1228-1236.

Dralle, K., Rudemo, M., 1997. Automatic estimation of individual tree positions from aerial photos. Canadian Journal of Forest Research 27, 1728-1736.

Fan, J., Gijbels, I., 1996. Local Polynomial Modelling and Its Applications. Chapman and Hall, London. 
Koppel, A., Oja, T., 1984. Regime of diffuse solar radiation in an individual Norway spruce (Picea abies (L.) Karst.) crown. Photosyntetica 18 (4), 529-535.

Larsen, M., 1997. Crown modelling to find tree top positions in aerial photographs. In: Proceedings of the Third International Airborne Remote Sensing Conference and Exhibition, Vol. II, Copenhagen, Denmark, ERIM International, pp. II-428-II-435.

Larsen, M., Rudemo, M., 1997. Using ray-traced templates to find individual trees in aerial photos. In: Proceedings of the 10th Scandinavian Conference on Image Analysis, Vol. 2, Lappeenranta, Finland, pp. 1007-1014.

Oker-Blom, P., Kellomäki, S., 1982. Theoretical computation of the role of crown shape in the absorbtion of light by forest trees. Mathematical Biosciences 59, 291-311.

Pollock, R.J., 1994a. A model-based approach to automatically locating individual tree crowns in high-resolution images of forest canopies. In: Proceedings of the First International
Airborne Remote Sensing Conference and Exhibition, Strasbourg, France.

Pollock, R.J., 1994b. A model-based approach to automatically locating tree crowns in high spatial resolution images. In: Desachy, J. (Ed.), Image and Signal Processing for Remote Sensing. Proc. SPIE 2315, Rome, Italy, pp. 526-537.

Pollock, R.J., 1996. The automatic recognition of individual trees in aerial images of forests based on a synthetic tree crown image model. Ph.D. Thesis, Computer Science, The University of British, Colombia, Vancouver, Canada.

Tibshirani, R., Hastie, T., 1987. Local likelihood estimation. Journal of American Statistical Association 82, 559-567.

Williams, D.L., 1991. A comparison of spectral reflectance properties at the needle, branch and canopy level for selected conifer species. Remote Sens. Environ. 35, 79-93.

Woodham, R.J., Gray, M.H., 1987. An analytic method for radiometric correction of satellite multispectral scanner data. IEEE Transactions on Geoscience and Remote Sensing 25 (3), 258-271. 